\title{
Hemangioma gigante del seno cavernoso. Caso clínico
}

\author{
M. Navas; M. Pedrosa-Sánchez; P. Martínez-Flórez; R. Carrasco; J.M. Pascual y R.G. Sola
}

Servicio de Neurocirugía. Hospital Universitario de la Princesa. Madrid. España.

Resumen

Introducción. El hemangioma intracraneal es una tumoración vascular benigna que raramente afecta al seno cavernoso, por lo que es frecuentemente diagnosticado de forma errónea como meningioma.

Caso clínico. Presentamos el caso de una mujer de 60 años que debutó con un cuadro de parálisis subaguda del tercer par craneal izquierdo. En neuroimagen, se evidenció una masa extraaxial localizada en la fosa craneal media, con extensión a las regiones selar y supraselar, e intenso realce homogéneo tras la administración de contraste. La arteriografía mostró discreto realce en fase arterial y venosa, $y$ efecto de masa. Se realizó una embolización del tronco meningohipofisario izquierdo.

Intervención y evolución. Se practicó una resección subtotal del tumor mediante un abordaje pterional izquierdo, persistiendo un pequeño resto intraselar. El diagnóstico anatomopatológico fue hemangioma. Tras la intervención la paciente no presentó nuevos déficits neurológicos.

Conclusión. El hemangioma debe ser tenido en consideración al realizar el diagnóstico diferencial de los tumores que afectan al seno cavernoso. Su tratamiento constituye un reto quirúrgico para el neurocirujano, dada la complejidad de esta región anatómica y la gran tendencia del tumor a sangrar durante su disección.

PALABRAS CLAVE: Hemangioma. Seno cavernoso.

Giant cavernous sinus haemangioma. Case report

Summary

Introduction. Intracranial haemangioma is a benign vascular tumor which seldom affects the cavernous sinus region, being it frequently misdiagnosed as a meningioma.

Recibido: 15-01-08. Aceptado: 28-02-08
Case report. A 60-year-old woman presented with a subacute-onset third cranial nerve palsy. A giant extraaxial mass located in the middle cranial fossa which extended into the selar and supraselar areas was diagnosed. It showed homogeneous enhancement after contrast administration. The angiography revealed mild enhancement as well as indirect signs of mass effect, and a tumoral embolization was performed. Surgery and outcome. The tumor was partially removed through a left pterional approach, leaving an intraselar remnant. The patient had an uneventful postoperative course, and did not present new neurological deficits.

Conclusions. The haemangioma must be considered in the differential diagnosis of cavernous sinus tumours. This entity represents a neurosurgical challenge, due to the complexity of this anatomical region and the trend of the tumour to bleed during its dissection.

KEY WORDS: Haemangioma. Cavernous sinus.

\section{Introducción}

Los hemangiomas son tumoraciones vasculares benignas de alto flujo, de localización variada y con una histología común. A nivel intracraneal, se sitúan con mayor frecuencia en el seno cavernoso, si bien pueden aparecer asociados a otros senos durales, incluyendo el seno petroso y la tórcula. Presentamos el caso de una paciente que fue intervenida en nuestro Servicio de un hemangioma gigante de seno cavernoso, realizando una revisión bibliográfica de las características clínicas y neurorradiológicas fundamentales, así como de las opciones terapéuticas de este tipo de lesión.

\section{Caso clínico}

\section{Presentación clínica}

Una mujer de 60 años fue remitida al Hospital de la Princesa para valoración neuroquirúrgica, tras ser diagnosticada de una lesión expansiva intracraneal. No destacaban antecedentes médico-quirúrgicos de interés, ni tratamien- 


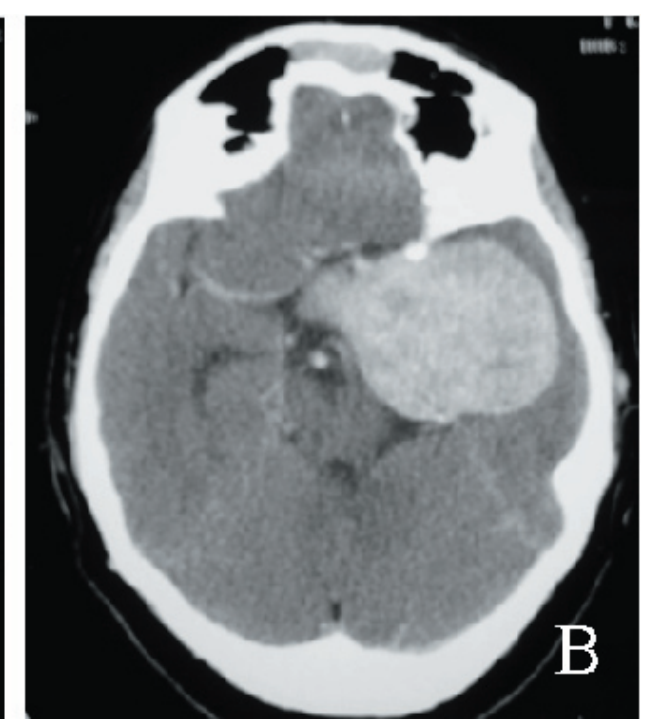

2009; 20: 461-466
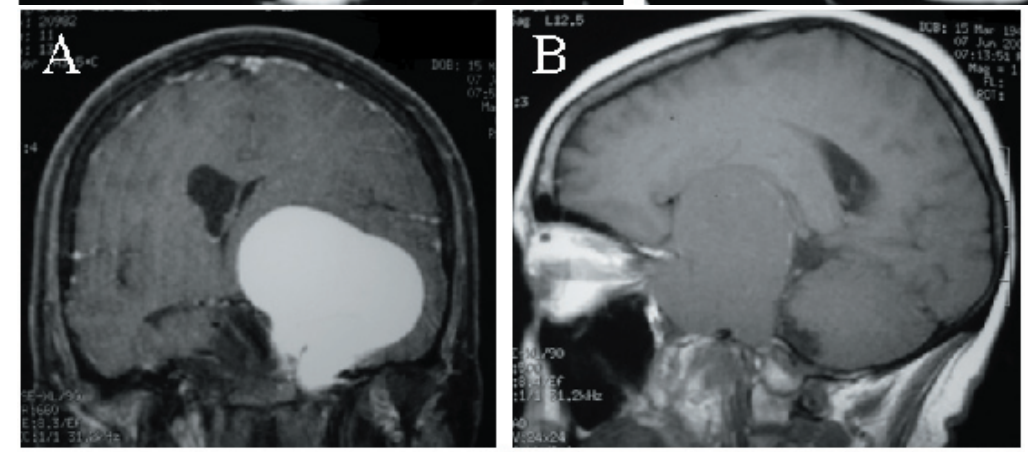
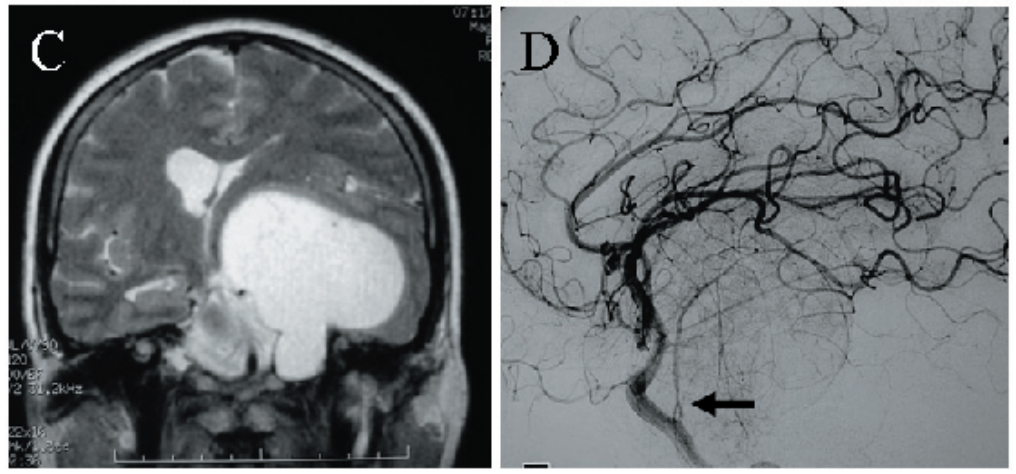

Figura 1. TAC cerebral. Se identifica una masa bien delimitada, hiperdensa, de características extraaxiales, que ocupa la fosa craneal media izquierda y se extiende hacia la silla turca $y$ el seno esfenoidal (A). Tras la administración de contraste, se produce un realce homogéneo de la lesión (B). tos farmacológicos en los últimos años. Refería, desde hace varios meses, episodios de desconexión del medio de unos segundos de duración. Asimismo, presentaba un cuadro de parálisis del tercer par izquierdo de quince días de evolución. En la exploración física se evidenció anisocoria, con una pupila izquierda midriática y arreactiva. La paciente no presentaba diplopia ni alteraciones en la agudeza visual. En el estudio endocrinológico destacó una leve hiperprolactinemia $(40,2 \mathrm{ng} / \mathrm{mL})$ y un cuadro de hipogonadismo hipofisario.

\section{Neuroimagen}

La TAC cerebral puso de manifiesto una lesión discretamente hiperdensa, localizada en la fosa craneal media, con expansión a las regiones selar y supraselar, sin edema vasogénico asociado (Fig 1).

El estudio de resonancia magnética (RM) evidenció una masa extraaxial de $64 \times 63 \times 51 \mathrm{~mm}$ a nivel del seno cavernoso izquierdo, con expansión a silla turca y seno esfenoidal (Fig 2). La lesión producía una discreta hiperostosis del ala menor esfenoidal y erosión de la punta del peñasco izquierdo. Angiográficamente, se evidenció una lesión con escaso realce en fases arteriales y venosas, y con efecto de masa sobre estructuras vasculares adyacentes (Fig 2). Se realizó una embolización del tronco meningohipofisario izquierdo con partículas de Polivinil Alcohol (PVA). El diagnóstico radiológico fue meningioma. 

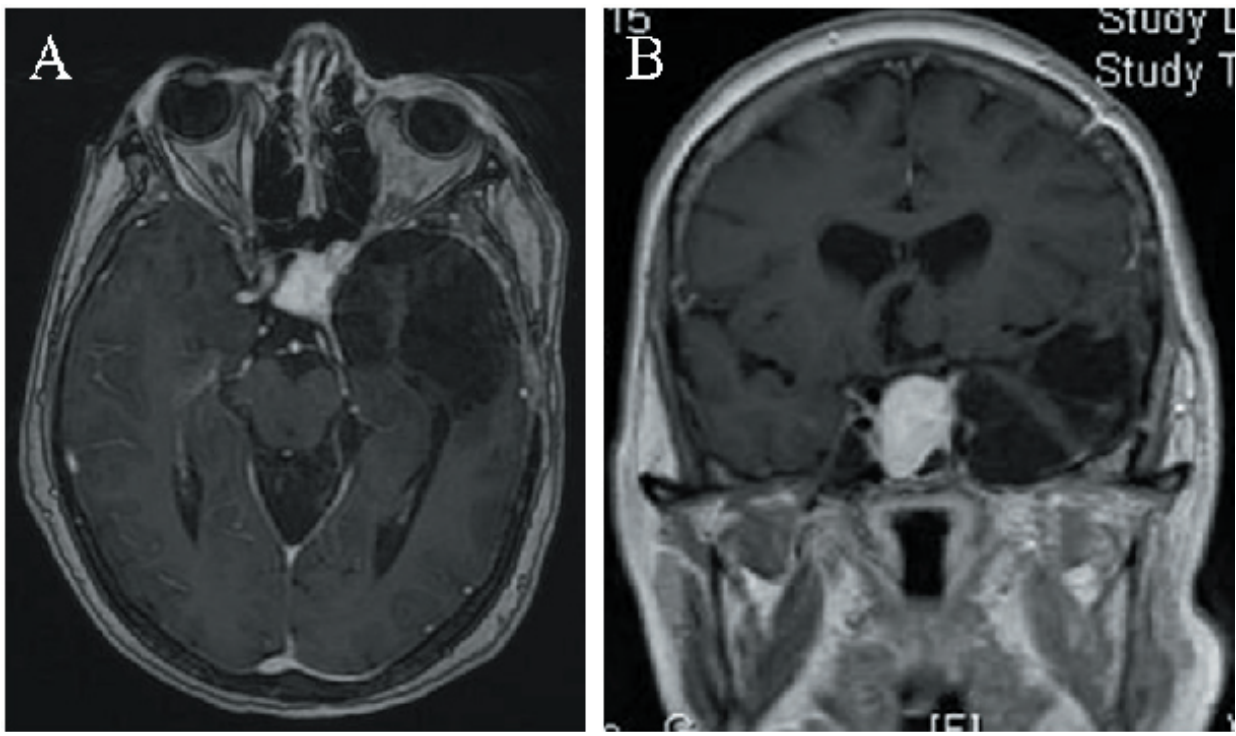

Figura 3. RM cerebral postquirúrgica con contraste. Cortes axiales (A) y coronales (B). Se aprecian áreas malácicas temporales izquierdas, así como un pequeño resto tumoral intraselar.

Intervención y evolución

La paciente fue intervenida mediante un abordaje pterional izquierdo, asistido por neuronavegación. A nivel de la fosa craneal media se identificó una gran tumoración extraaxial, muy vascularizada, de aspecto rojizo, la cual fue resecada en sus límites macroscópicos. Durante la intervención se produjo un importante sangrado de la lesión, que precisó la transfusión de dos concentrados de hematíes.

El estudio anatomopatológico fue informado como tumoración vascular de paredes finas e irregulares anastomosadas entre sí, hallazgos compatibles con hemangioma.

En el periodo postoperatorio inmediato, no se apreciaron nuevos déficits neurológicos. La paciente desarrolló una conjuntivitis en ojo izquierdo tratada con antibióticos tópicos, una trombosis venosa profunda de miembro inferior derecho que precisó tratamiento anticoagulante, así como un cuadro diferido de dolor hemifacial izquierdo, que respondió adecuadamente al tratamiento con carbamacepina.

A los seis meses de la intervención, la paciente se mantenía clínicamente estable. En la RM de control únicamente persistía un pequeño resto tumoral a nivel intraselar, que será tratado próximamente mediante radiocirugía.

\section{Discusión}

\section{Generalidades y epidemiología}

Los hemangiomas de seno cavernoso son lesiones extraaxiales poco frecuentes. Representan el 3\% de los tumores benignos y el $2 \%$ de los tumores localizados en esta región anatómica ${ }^{7,11,23}$. Tienen su origen en la arteria carótida interna intracavernosa, y adquieren su aporte vascular de ramas de la arteria meníngea media y de las ramas meníngeas accesorias ${ }^{2,7,11,17,23}$.

Sólo secundariamente, cuando aumentan de tamaño y se convierten en grandes (entre 3,1-4 cm) o gigantes (más de $4 \mathrm{~cm}$ ), se extienden e invaden la fosa craneal media. En la literatura existe cierta confusión a la hora de denominar estas lesiones. Se han referido a ellas de forma indistinta como malformaciones cavernosas, angiomas, hemangiomas de fosa craneal media o hemangiomas de seno cavernoso. La mayoría de los autores reservan el término angioma para referirse a lesiones vasculares malformativas. La denominación hemangioma se usaría para definir lesiones vasculares tumorales histológicamente benignas ${ }^{1,2}$. Entre el $80-95 \%$ aparecen en mujeres, con mayor incidencia en la población japonesa. Los síntomas se presentan entre los 30 y 60 años, si bien los pacientes más jóvenes suelen debutar con sintomatología más florida ${ }^{7,11,22}$. Los estrógenos podrían jugar un papel importante en la historia natural de estas lesiones. Se ha demostrado que durante el embarazo aumentan de tamaño, tornándose muchos de ellos sintomáticos o empeorando la clínica de los ya diagnosticados. En otros casos se produce involución después del parto, con una mejora de la sintomatología $a^{2,7,11,17,23}$.

\section{Presentación clínica}

A diferencia de las malformaciones vasculares, los hemangiomas no se asocian a sangrados espontáneos, si bien comprimen y distorsionan las estructuras adyacentes. Los pacientes suelen debutar con parálisis de pares craneales de instauración aguda o subaguda debido a la compresión de estructuras del seno cavernoso, como ocurrió en nuestra paciente. También pueden presentar cefalea, dolor o anestesia hemifacial o retroorbitaria, disminución de agudeza visual por compresión quiasmática, alteración del reflejo corneal e hipopituitarismo. La aparición de crisis comiciales no es frecuente.

No obstante, la sintomatología y los déficits neurológicos no son exclusivos de los hemangiomas, sino que son 
comunes para todos aquellos tumores que se originan o afectan secundariamente al seno cavernoso $0^{2,5,7,18,19,22,23}$.

\section{Diagnóstico radiológico}

En la TAC, los hemangiomas suelen aparecer como lesiones bien definidas, isodensas o hiperdensas en el estudio basal, que realzan de forma homogénea tras la administración de contraste, generalmente sin signos de hiperostosis adyacente a, $^{2723}$. En la RM son tumores característicamente hiperintensos en secuencias T2, con apariencia similar al líquido cefalorraquídeo (LCR), y realce homogéneo tras la administración de Gadolinio ${ }^{7,11,23}$. Estos datos pueden ayudar al diagnóstico preoperatorio de hemangioma, si bien muchos de ellos son considerados meningiomas antes de la cirugía, como sucedió en el caso que presentamos.

La angiografía es positiva en el $80 \%$ de los casos, con vascularización aferente dependiente del tronco meningohipofisario y de la arteria meníngea media. No obstante, la embolización prequirúrgica de la lesión no suele ser eficaz para disminuir el sangrado intraoperatorio ${ }^{7,11}$. En el restante $20 \%$, únicamente se aprecia una masa avascular con desplazamiento de estructuras adyacentes.

\section{Diagnóstico diferencial}

El 38\% de estas lesiones son diagnosticadas de forma errónea preoperatoriamente ${ }^{23}$. Los tumores benignos del seno cavernoso más frecuentes son los meningiomas, y hasta en un $75 \%$ de los casos pueden ser diferenciados de los hemangiomas $^{5,11}$. La presencia de cola dural, hiperostosis, calcificaciones, así como los datos aportados por la RM (más del $80 \%$ de los meningiomas son isointensos en secuencias $\mathrm{T} 2$, excepto los subtipos angioblástico y sincitial que realzan de forma intensa y homogénea) ayudan a discernir ambos tipos de lesiones ${ }^{11}$. Los neurinomas del trigémino tienen una apariencia muy similar a los hemangiomas en las secuencias T2 de la RM, si bien no presentan realce en el estudio angiográfico ${ }^{11}$. Además, suelen erosionar el agujero oval y se asocian clínicamente con una mayor tasa de neuralgia hemifacial. Otras lesiones que se deben incluir en el diagnóstico diferencial son aquéllas que pueden asentar en el seno cavernoso por extensión secundaria desde otras localizaciones, como los angiofibromas juveniles y los adenomas hipofisarios invasivos ${ }^{5,11,23}$.

\section{Histología}

Los hemangiomas son tumores benignos, constituidos por estructuras vasculares limitadas por endotelio. Carecen de componente muscular y presentan una pequeña cantidad de tejido elástico en la pared vascular, abundante contenido de colágeno y ausencia de signos de trombosis o calcificaciones. A menudo, se identifica una cápsula o pseudocápsula formada a partir de la duramadre. Algunos autores diferencian dos subtipos de hemangiomas en función de sus características histológicas ${ }^{19,22}$. Los de subtipo A son aquéllos formados por sinusoides de paredes finas, con escaso contenido en tejido conjuntivo, por lo que se colapsan de forma importante al manipularlos, pero presentan sangrados muy abundantes durante la cirugía. Los hemangiomas de subtipo B presentan sinusoides de paredes más gruesas, más cantidad de colágeno y una mayor consistencia, por lo que suelen sangrar menos, y esto facilita la resección completa del tumor. Aquellos hemangiomas que no presentan un realce homogéneo en el estudio de TAC, pertenecerán de forma más probable al subtipo $\mathrm{B} \mathrm{y}$, por tanto, se asociarán a un mejor control de la hemostasia ${ }^{11}$. Los datos aportados por la RM son menos útiles para predecir las características histológicas ${ }^{19}$.

\section{Tratamiento y pronóstico}

Wepfer (1620-1695), al estudiar el recorrido de la arteria carótida, fue el primer autor en definir el seno cavernoso. $\mathrm{Su}$ denominación actual se atribuye a Winslow, que en 1732 describió este espacio venoso trabeculado y angosto ocupado por la arteria carótida interna. Actualmente, se considera un entramado de plexos venosos que forma parte del sistema venoso extradural de la base del cráneo, desde la fisura orbitaria superior hasta el ápex del peñasco. Trescientos años después, gracias al exhaustivo conocimiento anatómico de esta región, ha sido posible acceder y tratar lesiones localizadas en el seno cavernoso ${ }^{7,9,17}$.

La cirugía se considera actualmente el tratamiento de elección de los hemangiomas de seno cavernoso, dada la naturaleza benigna de los mismos. El objetivo es realizar una resección tumoral completa preservando la máxima funcionalidad de las estructuras adyacentes, una meta especialmente complicada debido a la complejidad anatómica de esta región. Además, el riesgo de un sangrado intraoperatorio severo es elevado debido a la gran vascularización del hemangioma, lo que dificulta aún más el tratamiento quirúrgico de este tumor. En algunas series, la morbi-mortalidad asociada a la cirugía se sitúa alrededor de un $36 \%{ }^{1,6}$. Los hemangiomas hallados incidentalmente, de pequeño tamaño, sin una afectación importante de estructuras vásculonerviosas, no requieren tratamiento $\mathrm{y}$ pueden ser objeto de seguimiento clínico y radiológico. En algunos casos son intervenidos para filiar su origen. Sin embargo, aquéllos que se hacen sintomáticos deben ser tratados 22 .

Los abordajes extradurales, pterional y órbitozigomatico, son habitualmente empleados para acceder al seno cavernoso; algunos autores describen otros abordajes más novedosos (endoscópico transnasal-transesfenoidal) o menos utilizados (subfrontal, temporal) ${ }^{6,20}$. Mediante los abordajes extradurales al seno cavernoso se reduce la manipulación del lóbulo temporal $\mathrm{y}$, por lo tanto, disminuye el 
riesgo de presentar crisis comiciales postoperatorias. De igual forma, la adición de una osteotomía orbitozigomática permite una adecuada exposición del seno cavernoso y de la fosa craneal media, disminuyendo la necesidad de retracción cerebral ${ }^{6}$.

Debido a su disposición en el interior del seno cavernoso, el VI par craneal se asocia con una mayor tasa de lesión tras la cirugía. En cambio, el resto de pares craneales localizados a este nivel, (III, IV, V1,V2,V3) están protegidos por ambas hojas de duramadre que limitan las paredes lateral y medial del seno cavernoso, respectivamente; por ello, estos nervios se encontrarán distorsionados y desplazados por el tumor, pero es más sencillo identificarlos y respetarlos durante la cirugía. Es importante minimizar la manipulación de pares craneales durante la cirugía, sobre todo el III par, por lo que la mayoría de autores recomiendan acceder al tumor a través del triángulo lateral o de Parkinson (entre IV y V1) y el triángulo anterolateral o de Mullan (entre V1 y V2 ${ }^{4,6}$. La recuperación de la funcionalidad nerviosa tras la resección tumoral es variable; habitualmente es menor y más tardía cuanto mayor sea el tamaño del tumor. Si se realiza una resección completa, el porcentaje de recidivas es muy bajo. Sin embargo, dada la dificultad y la alta morbimortalidad derivadas de la misma, en ocasiones es preferible la extirpación subtotal y asociar tratamiento con radioterapia al resto tumoral existente ${ }^{20}$.

Dada la gran vascularización del tumor y el riesgo de sangrado intraoperatorio masivo, en algunos casos es posible realizar la resección del tumor tras el clipaje temporal de la arteria carótida interna ipsilateral; esto facilita su disección de estructuras vasculares adyacentes, reduce el sangrado de la lesión, y por tanto facilita la cirugía. Previamente al tratamiento quirúrgico, es necesario valorar la tolerancia del paciente a esta maniobra, mediante un test de oclusión. No obstante, se puede obtener un adecuado control de la arteria carótida interna accediendo a ésta a través del triángulo posteromedial o de Glascock, evitando de esta manera realizar clipajes temporales de la misma. En el caso que presentamos, se accedió a través de este espacio evitando la necesidad de interrumpir el flujo sanguíneo. Como consecuencia de la manipulación de la arteria carótida interna durante la cirugía, se han descrito casos de infartos cerebrales carotídeos ${ }^{5,6}$.

La radioterapia tiene un papel como tratamiento complementario tras la cirugía en los casos en que la resección tumoral no sea completa, así como para aquellos casos no resecables que fueron diagnosticados mediante biopsia. El mecanismo de acción de la radioterapia consiste en inducir una proliferación endotelial, la hialinización de las paredes vasculares y una posterior obliteración de los sinusoides9. De esta forma, aquellos hemangiomas diagnosticados mediante técnicas de neuroimagen, pueden beneficiarse del tratamiento con radioterapia previamente a la cirugía para reducir el sangrado intraoperatorio; este punto es de especial importancia, puesto que como se señaló previamente, la embolización preoperatoria de la lesión no reduce el sangrado quirúrgico en la mayoría de los $\operatorname{casos}^{22}$. Las dosis empleadas habitualmente se sitúan en torno a los 15-30 Gy. La mayor reducción en el tamaño tumoral se consigue con los primeros 30 Gy de tratamiento, siendo mínimos los cambios obtenidos posteriormente.

La radiocirugía es una opción terapéutica útil para la obliteración de lesiones vasculares intracraneales, y se asocia a una menor tasa de complicaciones en comparación con la radioterapia fraccionada ${ }^{14}$. Está indicado su uso para aquellos hemangiomas de pequeño tamaño que son diagnosticados por técnicas de neuroimagen o mediante biopsia, y en algunos casos de tumor residual tras la cirugía ${ }^{17}$.

\section{Bibliografía}

1. Anand, V.K., House, J.R., Al-Mefty, O.: Management of benign neoplasms invading the cavernous sinus. Laryngoscope 1991; 101: 557-564.

2. Biondi, A., Clemenceau, S., Dormont, D., et al: Intracranial extra-axial cavernous Hemangiomas: tumors or vascular malformations?. J Neuroradiol 2002; 29: 91-104.

3. Chen, J.C.T., Gianotta, S.L., Cheng, Y., Petrovich, Z., Levy, M.L., Apuzzo, M.L.J: Radiosurgical management of benign cavernous sinus tumors: dose profiles and acute complications. Neurosurgery 2001; 48: 1022-1032 .

4. Dolenc, V.V.: Transcranial epidural approach to pituitary tumors extending beyond the sella. Neurosurgery 1997; 41: 542-552.

5. Eisenberg, M.B., Al-Mefty, O., DeMonte, F., Burson, G.T.: Benign Nonmeningeal Tumors of the Cavernous Sinus. Neurosurgery 1999; 44: 949-954.

6. Goel, A., Muzumdar, D., Sharma, P.: Extradural approach for cavernous hemangioma of the cavernous sinus: experience with 13 cases. Neurol Med Chir (Tokyo) 2003; 43: 112-119.

7. Gonzalez, L.F., Lekovic, G.P., Eschbacher, J., Coons, S., Porter, R. W., Spetzler, R. F.: Are cavernous sinus hemangiomas and cavernous malformations different entities? Neurosurg Focus 2006; 21: 1-5.

8. Kanaan, I., Jallu, A., Alwatban, J., Patay, Z., Hessler, R.: Extra-axial cavernous hemangioma: two case reports. Skull Base 2001; 11: 287-295.

9. Kida, Y., Kobayashi, T., Mori, Y.: Radiosurgery of cavernous hemangiomas in the cavernous sinus. Surg Neurol 2001; 56: 117-122.

10. Kuo, J.S., Chen, J.C.T., Cheng, Y.: Gamma knife radiosurgery for benign cavernous sinus tumors: quantitative analysis of treatment outcomes. Neurosurgery 2004; 56 : 1385 1393.

11. Linksey, M.E., Sekhar, L.N.: Cavernous sinus 
hemangiomas: a series, a review, and an hypothesis. Neurosurgery 1992; 30: 101-107.

12. Mirzayan, M.J., Capelle, H.H., Stan, A.C., Goetz, F., Krauss, J.K.: Cavernous hemangioma of the cavernous sinus, skin and retina: hemodynamic changes after treatment: case report. Neurosurgery 2007; 60: E952.

13. Park, Ch.K., Lee, M.S., Kim, Y.G., Kim, D.H.: Cavernous hemangioma in the middle cranial fossa and cavernous sinus. J Korean Neurosurg Soc 2006; 40: 277-280.

14. Peker, S., Kihç, T., Şengöz, M., Pamir, M.N.: Radiosurgical treatment of cavernous sinus cavernous haemangiomas. Acta Neurochir (Wien) 2004; 146: 337-341.

15. Puca, A., Colosimo, C., Tirpakova, B., Lauriola, L., Di Rocco, F.: Cavernous hemangioma extending to extracranial, intracranial and orbital regions. J Neurosurg 2004; 101:10571060 .

16. Rhoton, A. L.: The cavernous sinus, the cavernous venous plexus and the carotid collar. Neurosurgery 2002; 51 (Suppl 1): 375-410.

17. Seo, Y., Furkuoka, S., Sasaki, T., Takanashi, M., Hojo, A., Kakamura, H.: Cavernous sinus hemangioma treated with Gamma knife radiosurgery: Usefulness of SPECT for diagnosis. Neurol Med Chir (Tokyo) 2000; 40: 575-580.

18. Shi, J., Wang, H., Hang, C., Pan, Y., Liu, C., Zhang, Z.: Cavernous hemangiomas in the cavernous sinus. Surg Neurol
1999; 52: 473-479.

19. Shi, J., Hang, Ch., Pan, Y., Liu, Ch., Zhang, Z.: Cavernous Hemangiomas in the Cavernous Sinus. Neurosurgery 1999; 45: 1308-1314.

20. Suri, A., Ahmad, F.U., Mahapatra, A.K.: Extradural transcavenous approach to cavernous sinus hemangiomas. Neurosurgery 2007; 60: 483-489.

21. Tannouri, F., Divano, L., Caucheteur, V.: Cavernous haemangioma in the cavernous sinus: case report and review of the literature. Neuroradiology 2001; 43: 317-320.

22. Thompson, T. P., Lunsford, L.D., Flickinger, J.C.: Radiosurgery for Hemangiomas of the Cavernous Sinus and Orbit: Technical Case Report. Neurosurgery 2000; 47: 778783.

23. Zhou, L.F., Mao, Y., Chen, L.: Diagnosis and surgical treatment of cavernous sinus hemangiomas: an experience of 20 cases. Surg Neurol 2003; 60: 31-38.

Navas, M.; Pedrosa-Sánchez, M.; Martínez-Flórez, P.; Carrasco, R.; Pascual, J.M.; Sola, R.G.: Hemangioma gigante del seno cavernoso. Caso clínico. Neurocirugía 2009; 20: 461-466.

Correspondencia postal: Marta Navas García. Hospital Universitario de la Princesa. C/Diego de León, 62. 28006, Madrid. 